\title{
Predesign of a flexible multibody system excited by moving load using a mechatronic system approach
}

\author{
Ghazoi Hamza ${ }^{1,2, *}$, Maher Barkallah ${ }^{1}$, Moncef Hammadi $^{2}$, Jean-Yves Choley ${ }^{2}$, Alain Riviere ${ }^{2}$, \\ Jamel Louati ${ }^{1}$, and Mohamed Haddar ${ }^{1}$ \\ ${ }^{1}$ Mechanics Modeling and Production Research Laboratory (LA2MP), National School of Engineers of Sfax (ENIS), \\ University of Sfax, B.P, 1173, 3038 Sfax, Tunisia \\ ${ }^{2}$ QUARTZ EA 7393, SUPMECA, 3 rue Fernand Hainaut, 93407 Saint-Ouen Cedex, France
}

Received: 24 April 2018 / Accepted: 25 August 2020

\begin{abstract}
In this paper, a new analytical approach to object oriented modeling is presented for the predesign of a multibody system. We investigate the dynamic behavior of a system of interconnected components using the modeling language Modelica. In engineering, beam-masses are often used as design models. In fact, the considered system is composed of a flexible beam subjected to a moving load and supporting one or more translating elastic subsystems. Each subsystem is controlled by a vibration absorber and the structure is affected dynamically only through the moving character of the load. The problem of calculation of the dynamic response of this system is very important in many engineering applications such as in the predesign and analysis of a robotic portal systems, machine tools and bridge crane systems. The object oriented modeling approach will be presented to demonstrate the importance of this approach to parametric investigation. It will illustrate how subsystems from Modelica Standard Library can be connected to the developed structure in order to study the vibrational behavior of such a system. For validation purpose, results are compared with those reported in the literature.
\end{abstract}

Keywords: Predesign / flexible multibody system / analytical approach / vibration

\section{Introduction}

Multibody dynamic analysis represents a powerful tool to investigate the dynamic response of flexible systems of arbitrary complexity and topology $[1,2]$. The multibody system modeling is a common approach for representing mechanical systems as interconnected components $[3,4]$. In fact, a flexible multibody system can be composed of elastic and rigid components which are connected by force and/or joints elements as springs, dampers, and actuators [5,6]. Alessandro et al. [7] propose two new Modelica libraries which are the Engines libraries and the Powertrain Dynamics (PTDynamics). The libraries enable the modeling and the simulation of a multibody system in one simulation environment (Dymola) based on Modelica language. Indeed, Modelica is very appropriate for building muli-domain models [8]. Indeed, Modelica contains many libraries which help the user in constructing multi-domain models.

\footnotetext{
* e-mail: hamza.ghazoi@gmail.com
}

The skeleton of a mechatronic system is the mechanical part. Mechanical systems may be conceptualized as elastic and/or rigid bodies. Schneider et al. [9] studied the dynamic and static behavior of a 5-AxisMilling Machine. The mechanical model of the machine is implemented in Modelica with rigid and flexible bodies. In fact, using the object oriented modeling approach; a mathematic representation of a physical model may be structured to get a reusable model that is easy to understand and to modify.

In the predesign phase, investigating the physical interactions between the diverse subcomponents of a mechatronic system is of great importance [10]. At this stage, a preliminary description of a mechanical system is essential for different constraints such as vibration, temperature, etc. Each physical phenomenon can be described by a mathematical model. Thus, a mechanical system modeling and simulation which may be based on numerous mathematical models play an important role in order to understand how the properties and the performances of the mechanical components and subsystems have an effect on the overall mechatronic system design [11-13]. 
The presence of vibrations is one of the main causes that limit performance in mechanical systems. The study of the dynamic behavior is having a great importance in the field of mechanical, aerospace engineering and civil [14-17]. For instance, in the field of engineering, a variety of structure is subjected to moving load. External forces, masses or whole subsystems moving with constant or varying speed along the structure can be the cause of these loads. Bridges, cranes and railway track are some cases of structures subjected to dynamic load. The study of the structure's reaction to these moving loads is very essential. Moreover, the dynamic of flexible structures with moving loads have been the subjects of many studies. For example, Zrnic et al. [18] studied the impact of a moving load to dynamic behavior of container cranes. The dynamic interaction between the supporting structure and the trolley caused by the moving load is also investigated.

There are many engineering problems can be modeled as the coupled structure-simple degree of freedom (SDOF) system, such as a motor, engine and electronic card elastically mounted on a structural element. For instance, Hamza et al. [19] proposed a predesign method applied to a mechatronic system in order to study its vibrational effect. The interaction between different subsystems (motors and electronics cards) located on a simply supported plate has been investigated. The electronic card is modeled as a simple degree of freedom system attached to the main structure (the plate) and the motor is modeled as a dynamic excitation over a finite area on the plate.

Vibration control is very important in order to reduce structural vibrations. Structural control systems are classified into two main categories which are passive and active control system. Dynamic vibration absorber (DVA) is one of the techniques used in vibration control. It consists of attaching a secondary mass to a primary vibrating system such that the secondary mass dissipates the energy of the primary system and reduces its vibration amplitude.

When machinery systems are subjected to vibrations due to external disturbances, vibration absorbers have been used widely for vibration reduction. Lin and Cho [20] proposed the application of a DVA in order to reduce the excessive vibration of a simply supported beam traversed by multiple moving loads. Soares et al. [21] studied the dynamic response of an Euler-Bernoulli beam subjected to moving loads and controlled by a moving vibration absorber. The vibration absorber is modeled as a linear spring-mass-damper system moving with a constant speed along the beam. The importance of speed and position of the damper on the vibration control of the beam subjected to moving loads is shown in this paper. To suppress the excessive vibration of a beam under a moving load, Sung [22] employed piezoelectric actuators that are bonded at the bottom of the beam at different positions.

The dynamic vibration absorber is probably the most common device for passive vibration of machines. It can be also called tuned mass damper (TMD). Because it is simple and robust, it has been used in many applications such as mechanical, civil and aerospace engineering. For instance, Chaari [23] proposed a passive vibration absorber model in order to decrease the vibration amplitude of a milling tool. Samani et al. [24] studied the performances of different

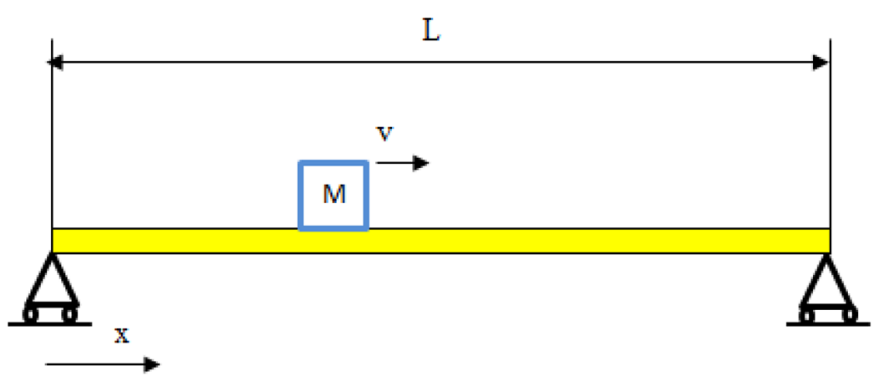

Fig. 1. The beam model subjected to a moving load.

types of dynamic vibration absorbers. A (DVA) is connected to a simply supported beam in order to reduce the excessive vibrations. The beam is traversed by moving loads/vehicles. The vehicle is modeled by a single degree of freedom mass spring system. To find the optimal parameters of the DVA that minimize the beam vibration, a parametric analysis is performed.

In the present study, a new mechatronic-based approach to model and simulate a multibody system is presented. We study the structure interacting through moving load using Modelica language. The beam structure carried one or more subsystems which are modeled as simple degree of freedom systems. The excessive vibration of the subsystem is controlled using a dynamic vibration absorber. The paper is organized as follows: the governing equations of a flexible beam and a connected subsystem will be derived in Section 2. Section 3 presents the methodology for modeling and control design of such multibody system. Numerical examples of the proposed methodology appear in Section 4. Section 5 concludes the paper.

\section{Multibody formulation}

The theoretical background of multibody systems is described in this section. The equations of motion are expressed as differential-algebraic equations (DAE).

\subsection{Model formulation of the beam vibration}

The system under consideration is shown in Figure 1. Consider a moving mass rolling on a finite simply supported beam at a specified speed. The Euler-Bernoulli beam theory is used, ignoring shear deformation effects. The beam is considered as a linear elastic continuous system. The effect of damping was considered negligible. The Fourier approach used in [25] will be adopted in our study.

The equation of motion for an Euler-Bernoulli beam under a general forcing function can be written as [25]:

$$
E I \frac{\partial^{4} u(x, t)}{\partial x^{4}}+[\rho S+M \delta(x-v t)] \frac{\partial^{2} u(x, t)}{\partial t^{2}}=g M \delta(x-v t) .
$$

In equation (1), $u$ is the displacement of beam in the y direction, $S$ is the cross-sectional area, $l$ is the beam length, $E I$ is the bending stiffness, $t$ is the time, $\rho$ is the mass density, $M$ is the mass of the moving load and $v$ is the load speed over the beam. 
$\delta(x-v t)$ is the Dirac Delta function

$$
\delta(x-v t)=0 \quad \text { for } \quad x \neq v t .
$$

The boundary conditions of a simply supported beam could be defined as:

$$
u(0)=0, \frac{d^{2} u}{d x^{2}}(0)=0, u(l)=0, \frac{d^{2} u}{d x^{2}}(l)=0
$$

The initial conditions of the problem are given by,

$$
u(x, 0)=0, \frac{\partial u}{\partial t}(x, 0)=0 .
$$

The beam is simply supported at both ends; the Eigenfrequency for $n$th mode is given by:

$$
\omega_{i}=\frac{i^{2} \pi^{2}}{l^{2}} \sqrt{\frac{E I}{\rho A}}
$$

In addition,

$$
\int_{0}^{l} \delta\left(x-v_{t}\right) d x=1 .
$$

Applying the Fourier finite sine transform,

$$
F(m, t)=\int_{0}^{l} u(x, t) \sin \frac{m \pi x}{l} d x .
$$

The transformed equation of the problem is [25],

$$
\begin{aligned}
F_{t t}(m, t)+ & \omega_{m}^{2} F(m, t)+\frac{1}{\rho S L} M \\
& \times\left[F_{t t}(m, t)+2 \sum_{k=1}^{\infty} F_{t t}(k, t) \sin \frac{k \pi v t}{l} \sin \frac{m \pi v t}{l}\right] \\
& =\frac{g}{\rho S} M \sin \frac{m \pi v t}{l} .
\end{aligned}
$$

We consider only the linear inertia term, equation (8) becomes [25]:

$$
F_{t t}(m, t)+\frac{\omega_{m}^{2}}{(1+R)} F(m, t)=\frac{P}{(1+R)} \sin \frac{m \pi v t}{l}
$$

With,

Then,

$$
R=\frac{M}{\rho S l}
$$

$$
\begin{aligned}
F(m, t) & =\frac{p}{\omega_{m}^{2}\left[1-\left[\frac{m \pi v \sqrt{1+R}}{l \omega_{m}}\right]^{2}\right]} \\
& \times\left[\sin \frac{m \pi v t}{l}-\frac{m \pi v \sqrt{(1+R)}}{l \omega_{m}} \sin \frac{\omega_{m} t}{\sqrt{(1+R)}}\right]
\end{aligned}
$$

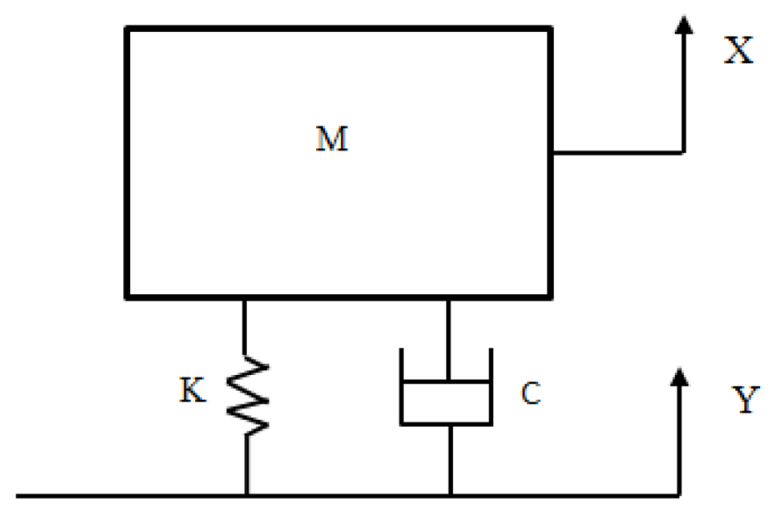

Fig. 2. Subsystem parameters.

Defining,

$$
P=\frac{M g}{\rho S}
$$

The expression of the beam displacement $u(x, t)$ can be assumed as [25]:

$$
\begin{gathered}
u(x, t)=\frac{2 P}{l} \sum_{m=1}^{\infty} \frac{\left[\sin \frac{m \pi v t}{l}-\frac{m \pi v \sqrt{1+R}}{l \omega_{m}} \sin \frac{\omega_{m}}{\sqrt{(1+R)}} t\right]}{\omega_{m}^{2}\left(1-\left(\frac{m \pi v \sqrt{(1+R)}}{L \omega_{m}}\right)^{2}\right)} \\
\sin \frac{m \pi x}{l} .
\end{gathered}
$$

\subsection{Modeling of the connected subsystem}

This section shows the dynamic behavior of a subsystem attached to the flexible beam. It is modeled as an oscillator model (a single degree of freedom mass spring system) on contact with the beam. The connected subsystem vibrates as a result of the oscillation of the support structure. The body mass is small compared to the beam mass, the effect of the beam on the subsystem in only taken into account. Figure 2 shows a simple representation of the subsystem model.

The balance equation of the subsystem is:

$$
\ddot{Z}+2 \sigma \xi \dot{Z}+\sigma^{2} Z=-\ddot{Y}
$$

$Z$ is the relative displacement of the subsystem with respect to the beam.

With,

$$
Z=X-Y
$$

where $\dot{Z}$ and $Z$ are respectively, the relative velocity and acceleration of the subsystem. $X$ is the absolute displacement of the subsystem and $Y$ is the base input displacement. 
The input displacement is expressed as follows:

$$
\begin{aligned}
& Y\left(x_{1}, t\right)=u\left(x_{1}, t\right)=\frac{2 P}{l} \sum_{m=1}^{\infty} \\
& \times \frac{\left[\sin \frac{m \pi v t}{l}-\frac{m \pi v \sqrt{1+R}}{l \omega_{m}} \sin \frac{\omega_{m}}{\sqrt{(1+R)}} t\right]}{\omega_{m}^{2}\left(1-\left(\frac{m \pi v \sqrt{(1+R)}}{L \omega_{m}}\right)^{2}\right)} \sin \frac{m \pi x_{1}}{l}
\end{aligned}
$$

with $x=x_{1}$ defines the location of the subsystem in the beam at time t. $\sigma=\sqrt{\frac{K}{M}}$ is the angular frequency, $M$ is the moving mass and $K$ is the spring stiffness.

The loss factor $\xi=\frac{C}{C_{c}}$ with, $C$ is the viscous damping coefficient and $C_{c}=2 M \sigma$.

The damped natural frequency,

$$
\omega_{d}=\sigma \sqrt{1-\xi^{2}}
$$

\section{Modeling and control of the multibody system}

\subsection{Problem statement}

We consider a flexible beam structure subjected to a moving load and carrying one body (SDOF system). The application of a damper absorber is proposed to reduce the excessive vibration of the subsystem which can be conducive to failure because of fatigue. The absorber may absorb passively a major portion of the vibration energy of the attached body induced by the moving load, hence acting as an energy dissipator. The damped vibration absorber is considered as a physical mechanical device. It is represented by a linear spring-mass-damper system connected to the subsystem. These parameters are the absorber mass $M_{A}$, the absorber viscous damper coefficient $C_{A}$ and the absorber spring stiffness $K_{A}$, as presented schematically in Figure 3 . In this system, $X_{1}$ corresponds to the displacement of the subsystem and the coordinate $X_{a}$ corresponds to the displacement of the absorber mass $M_{A}$. Choosing the correct parameters of the absorber is very important in order to dissipate disturbances of the subsystem [26].

\subsection{Modelica implementation}

The motivation of using Modelica is the object-oriented language characteristic for modeling complex, large, and heterogeneous physical systems. It is designed to support library development. For handling complexity, reuse is a key issue. Algebraic, differential and discrete equations are used in Modelica for modeling the physical phenomena.

A flexible multibody system has been developed in Modelica using the Dymola simulation environment

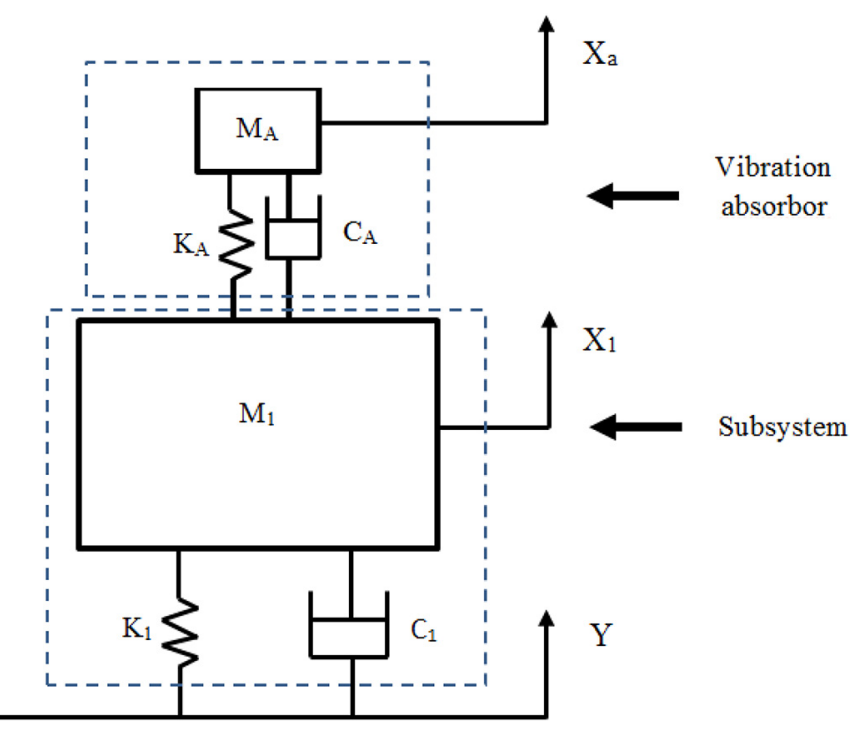

Fig. 3. Simple vibrating subsystem with DVA.

(see Fig. 4). The system model includes elements developed specifically for this application such as the Beam component and the Excitation source component as well as parts that belong to Dymola/Modelica library. The system is then comprised of a flexible beam, an excitation source, a subsystem, a vibration absorber and sensors. The interaction between the different components is described using connectors.

The beam element is developed in-house based on the analytical model formulation presented in Section 2 . The Beam model is parameterized such that the user has the ability to easily enter the beam characteristics. Its parameters include the Young's modulus $E$, the beam length $l$ and the mass density $\rho$, etc. It has been implemented with two point connectors. Excitation source object represent a vibration source moving along the flexible beam with constant magnitude and velocity. The user has the ability to modify its parameters.

The beam is mechanically connected to the excitation source. We have developed a new connector in order to connect the excitation source to the flexible beam. The code of the new connector is:

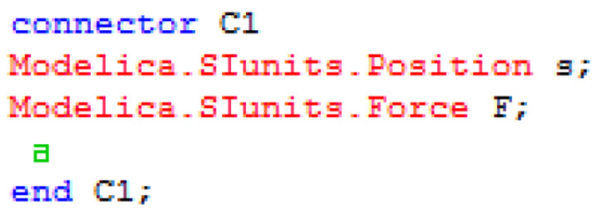

The Beam component contains a Flange type connector (as it is called in Modelica). The choice of Flange connector makes the beam component fully compatible with the Modelica Translational library elements. It is possible then to directly connect the flexible beam component to the predefined models, such as force elements (springs, dampers, and forces). This approach confirms that this model is a reusable component and can be used as a building block in other applications as well. 


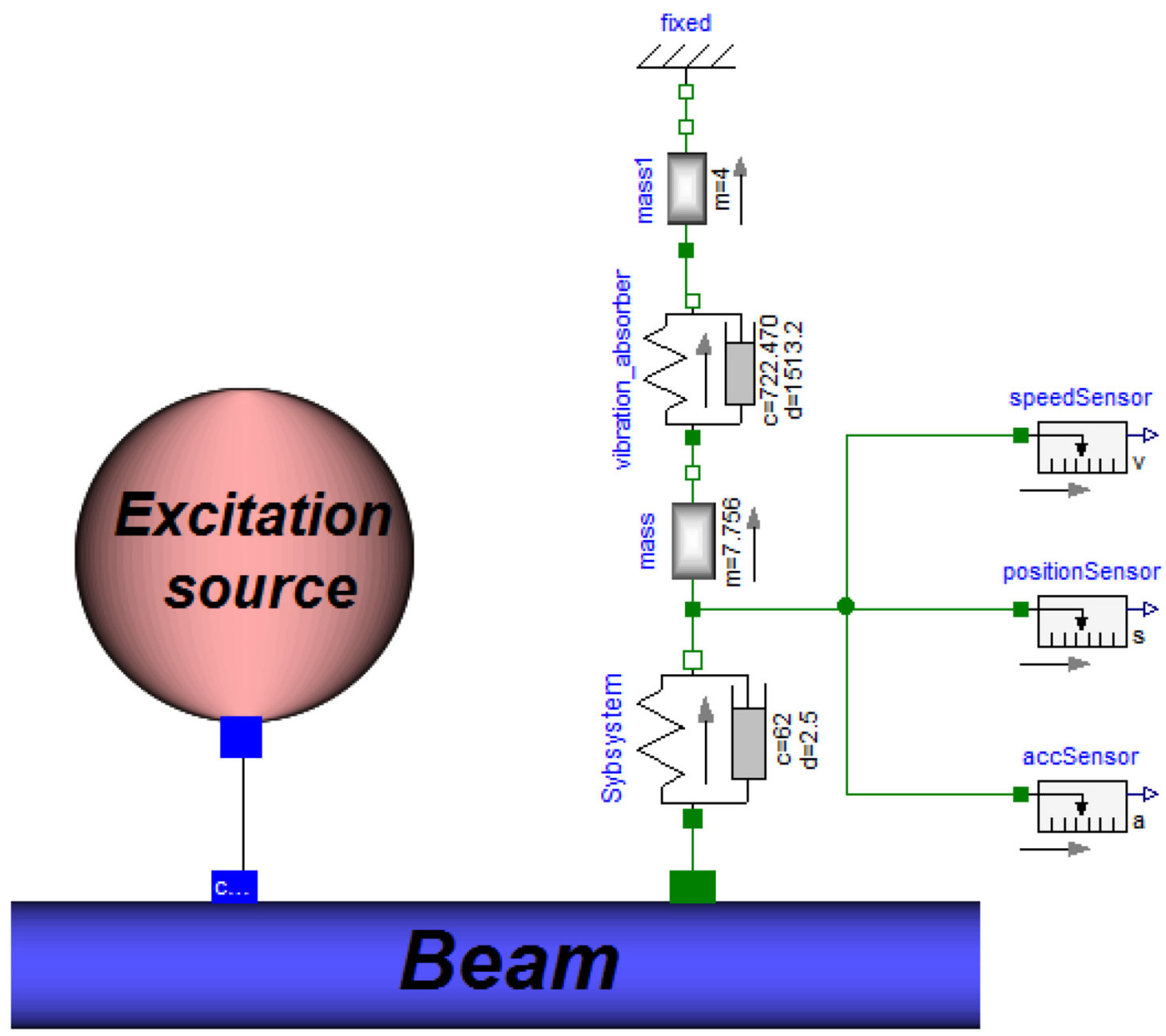

Fig. 4. Model of the system in Modelica/Dymola.

In the developed system model, the beam is connected to a subsystem which belongs to the Modelica Standard Library (MSL). It consists of a linear 1D model composed of three parts which are a spring $\mathrm{K} 1$ and damper $\mathrm{C} 1$ in parallel and a concentrated mass M1. The subsystem can be located at an arbitrary placement along the beam chosen by the user.

The subsystem is connected to a dynamic vibration absorber in order to attenuate the excessive vibrations. The DVA is modeled as a single degree of freedom system composed of three parts which are a spring $\mathrm{K}_{\mathrm{A}}$ and damper $\mathrm{C}_{\mathrm{A}}$ in parallel and a concentrated mass $M_{A}$.

We have implemented sensors in order to measure the vibration response of the subsystem on the beam (absolute position, absolute velocity and absolute acceleration).

These component models are used to build a complete system model. In this approach, mechanics together with controller dynamics are designed simultaneously in order to obtain the characteristics of the vibrational motion of the multibody system. In order to design more efficient system for more accurate specifications, this procedure simplified the problem complexity because the effect of the change in the system properties such as beam length is easily accommodated in this problem.
Table 1. Details of the test beam.

\begin{tabular}{ll}
\hline Property & Value \\
\hline Young modulus & $E=2.117 \times 10^{11} \mathrm{~N} / \mathrm{m}^{2}$ \\
Mass density & $\rho=8000 \mathrm{~kg} / \mathrm{m}^{3}$ \\
Beam length & $l=10 \mathrm{~m}$ \\
Cross section & $A=16 \times 10^{-4} \mathrm{~m}^{-4}$ \\
\hline
\end{tabular}

\section{Results and discussion}

\subsection{Validation}

In order to check the accuracy of the present model, some results obtained by Modelica/Dymola are compared with those available in the literature. The parameters used in the simulation are shown in Table 1.

Table 2 lists the first three natural frequencies along with analytical results and those obtained by the finite element method used Matlab code [27]. In [27], authors used fifty identical finite elements in order to study the dynamic behavior of a beam carrying a moving load. 
Table 2. Natural frequencies of the simply supported beam without mass $(\mathrm{Hz})$.

\begin{tabular}{lll}
\hline Mode & $\begin{array}{l}\text { FEM (Matlab) } \\
{[26]}\end{array}$ & $\begin{array}{l}\text { Analytical method } \\
\text { (Dymola) }\end{array}$ \\
\hline 1 & 0.9330 & 0.933 \\
2 & 3.7319 & 3.7322 \\
3 & 8.3959 & 8.397 \\
\hline
\end{tabular}

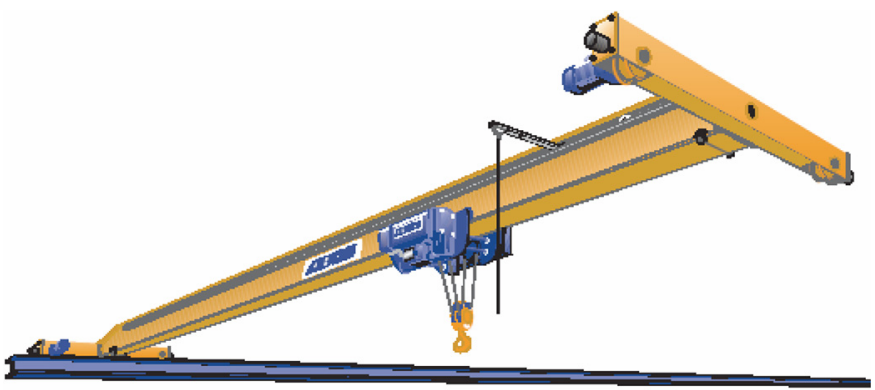

Fig. 5. Bridge crane system [25].

Table 3. Parameters used in the dynamic simulation of the system.

\begin{tabular}{ll}
\hline Property & Value \\
\hline Young modulus of elastic beam & $E=2.1 \times 10^{11} \mathrm{~N} / \mathrm{m}^{2}$ \\
Mass density of elastic beam & $\rho=7850 \mathrm{~kg} / \mathrm{m}^{3}$ \\
The girder length & $l=40 \mathrm{~m}$ \\
Cross section & $A=0.04 \mathrm{~m}^{2}$ \\
$\begin{array}{l}\text { Area moment of inertia } \\
\text { of elastic beam }\end{array}$ & $I=0.00667 \mathrm{~m}^{4}$ \\
$\begin{array}{l}\text { Speed } \\
\text { The mass of the payload } \\
\text { and trolley }\end{array}$ & $v=2 \mathrm{~m} / \mathrm{s}$ \\
\hline
\end{tabular}

The beam is made of steel and square in cross section. The accelerating mass is modelled as a moving finite element to include gravitation force and inertial effects of mass.

The natural frequencies obtained by the two models are closely the same. Results are then in good accordance.

For verification of the proposed model, an example of a bridge crane system is considered in this paper. The mechanism consists of a trolley moving over a girder (Fig. 5). The trolley moves at a constant speed. The support structure is modeled as a simply supported beam. The structure is dynamically affected through the moving character of the trolley only. The mass of the moving substructure is composed of the mass of trolley and the mass of payload, which are modeled as a lumped mass.

The geometrics and materials properties used in this simulation are summarized in Table 3 .

The validity of the model is verified by comparing the dynamic response of the adopted analytical model with

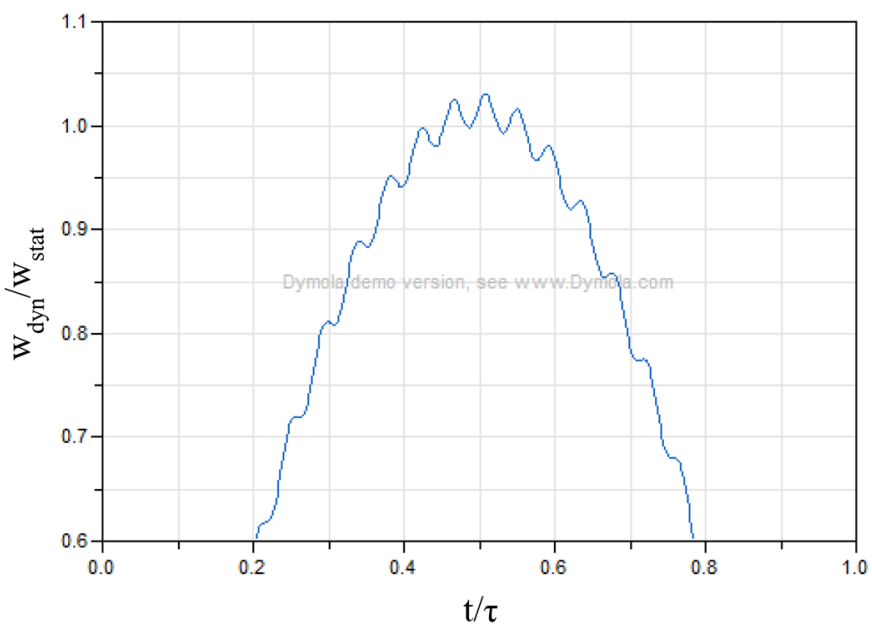

Fig. 6. Central response of the simply supported beam due to moving mass.

Table 4. System parameters [29].

\begin{tabular}{ll}
\hline Property & Value \\
\hline Mass of the attached body & $M_{1}=7.756 \mathrm{~kg}$ \\
Spring stiffness & $K_{1}=62,000 \mathrm{~N} / \mathrm{m}^{3}$ \\
Damper & $C_{1}=2.500 \mathrm{~N} \mathrm{~s} / \mathrm{m}$ \\
Absorber mass & $M_{A}=4 \mathrm{~kg}$ \\
Absorber spring stiffness & $K_{A}=722.470 \mathrm{~N} / \mathrm{m}^{3}$ \\
Absorber viscous damper & $C_{A}=1513.2 \mathrm{~N} \mathrm{~s} / \mathrm{m}$ \\
coefficient & \\
\hline
\end{tabular}

those obtained in the example presented in [28] using the finite element method. In Figure 6, the dynamic beam displacement response curve predicted by the analytical model coincides well with those in Figure 8 [28] which confirms the reliability of the presented theory.

\subsection{Parametric study}

In this section, the effects produced by the variation of physical parameters to the response of the system are examined. In all calculations, the following values are used: $l=20 \mathrm{~m}, A=0.04 \mathrm{~m}^{2}, \rho=7850 \mathrm{~kg} / \mathrm{m}^{3}$ and $I=0.00667 \mathrm{~m}^{4}$. The beam is subjected to an overall mass of $10000 \mathrm{~kg}$, moving with constant speed $v=4 \mathrm{~m} / \mathrm{s}$.

The physical parameters of the attached body and the associated dynamic vibration absorber used in the simulations are listed in Table 4 . The proposed model allows the designer to perform a simultaneous simulation of the mechanical system and the controller.

The effect of the application of the vibration absorber to suppress the excessive subsystem vibration is shown in Figure 7. This figure shows the maximum displacement of the subsystem with and without vibration absorber with zero initial conditions. The subsystem is concentrated at the middle span of the beam. As it is shown, the inclusion of the absorber decreases the maximum displacement of 


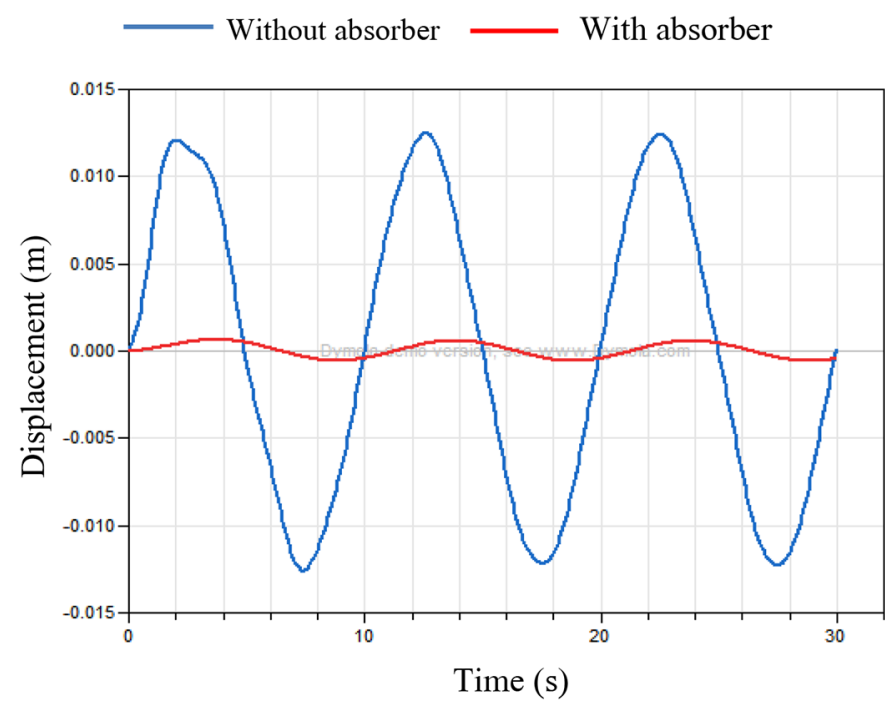

Fig. 7. Comparison maximum displacement of the subsystem.

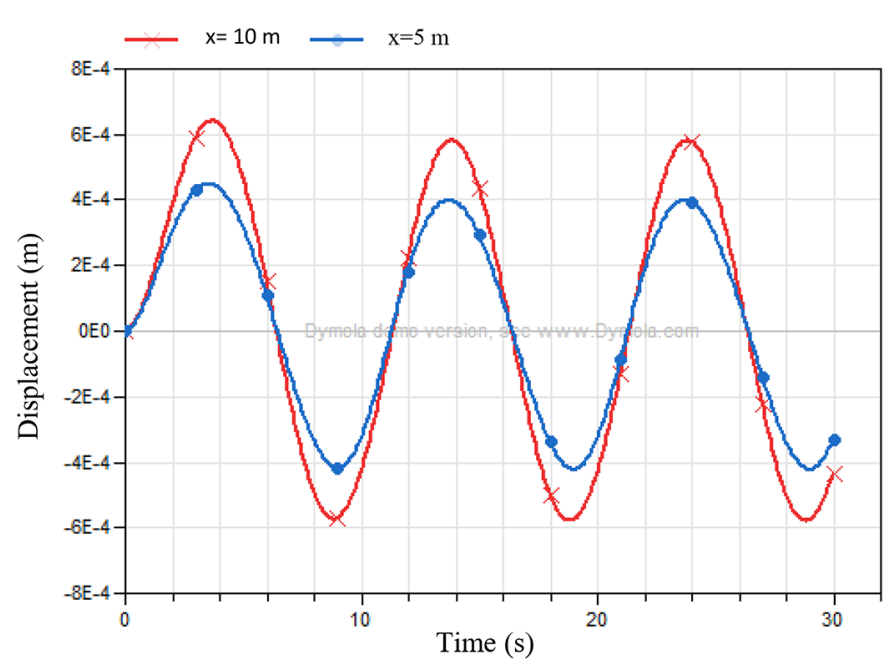

Fig. 8. Subsystem response.

the subsystem which is connected to the beam. The effects of the different design parameters of the absorber (the stiffness, the viscous damping coefficient) can be examined to find the optimum values. Using this model, the designer has the ability to directly access to structural and control parameters.

The change in position of the concentrated mass has a significant effect on its motion. Figure 8 shows the displacement of the subsystem with absorber located at $x=5 \mathrm{~m}$ and $x=10 \mathrm{~m}$ from the left end of the beam. As expected, the vibration amplitude is more important when the subsystem is located in the mid span of the beam.

Figure 9 indicates the displacement of the beam mid span versus the position of the load along the beam. The maximum value of deflection is obtained to be equal $0.012 \mathrm{~m}$, when the moving load is in the mid span of the beam.

The influence of speed on the dynamic response of the system is investigated. The responses of the beam where

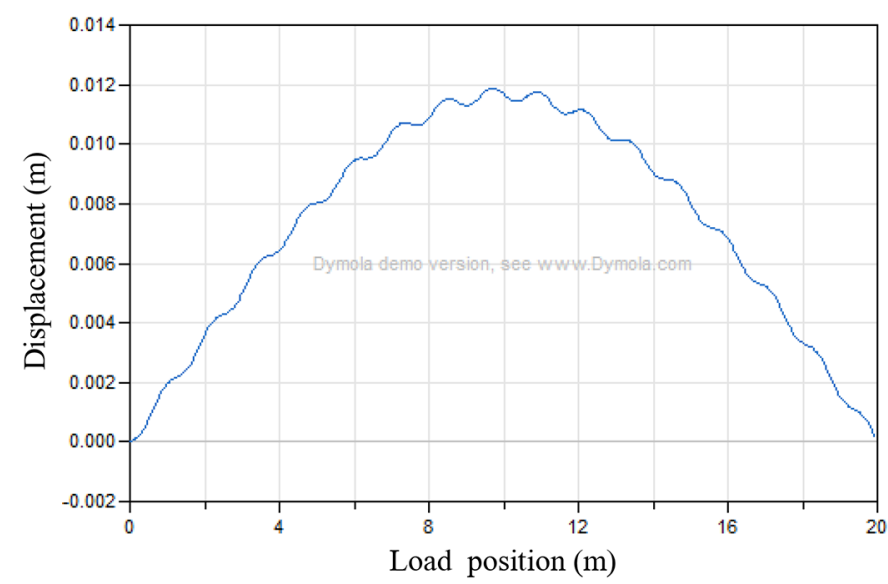

Fig. 9. Beam deflection according to the moving load position.

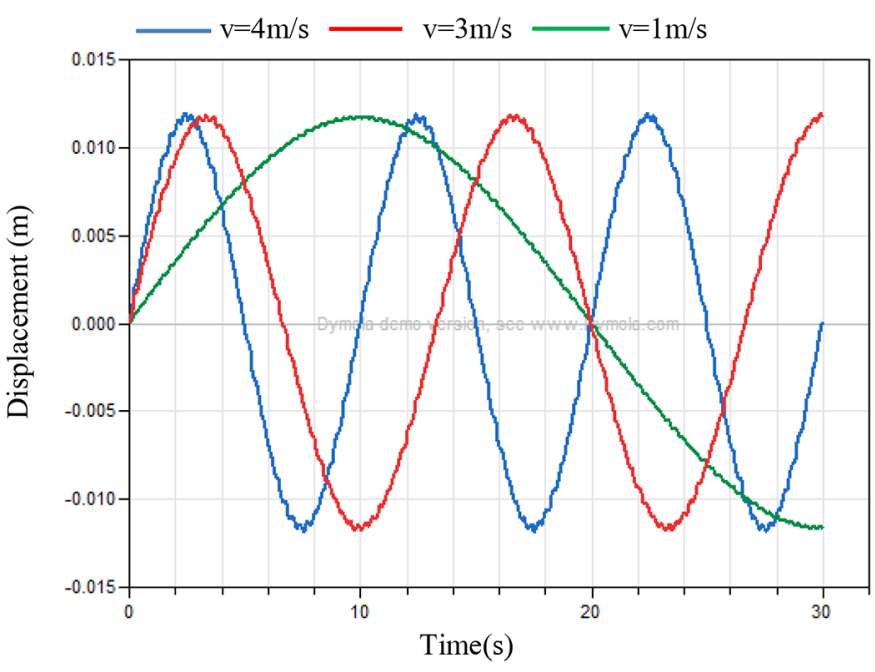

Fig. 10. Vertical beam deflections for various speeds.

plotted for various speed values for the moving load. As it is apparent in Figure 10, the more the speed increases the more the deflection curve shape tends to the left end. This is due to the coupling between the beam motion and the motion of the load. Thus, the maximum point depending on the increasing speed travels to the right end of the beam.

Figure 11 depicts the beam responses for various load masses. It is evident from the graph that the defection magnitude increases with the mass of the load.

The proposed model allows the designer to predict the system dynamic response and the control action and to evaluate the performance of the multibody system.

\section{Conclusion}

In this paper, a new predesign approach to study the flexibility influence of a multibody system on its dynamic behavior is presented. To obtain quick resolutions, analytical method is approached to study the system response. Analytical models are developed using the object oriented modeling approach with Modelica language. 


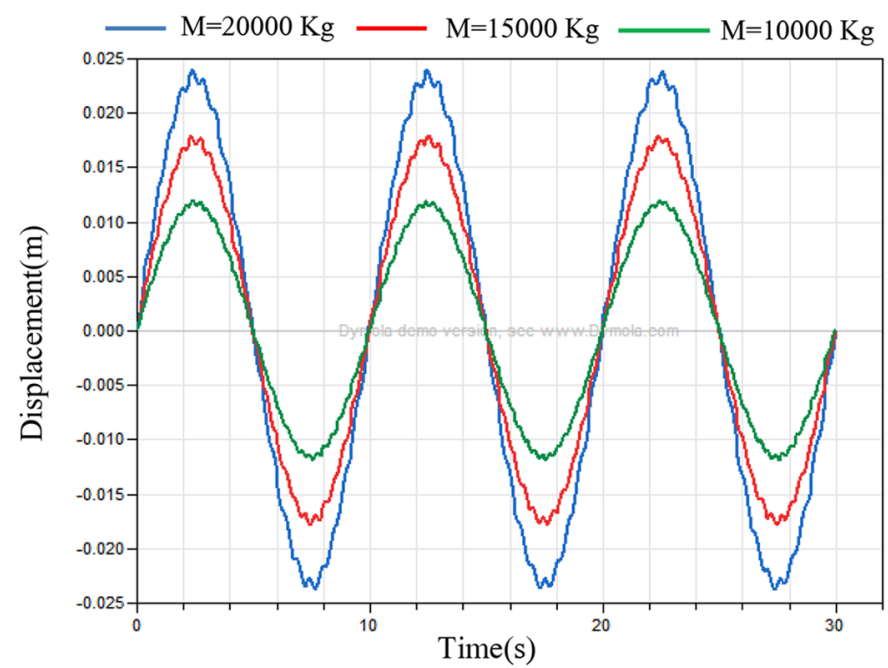

Fig. 11. Dynamic response at the beam center for various load masses.

The developed models use standard connectors to ensure the compatibility with Modelica Standard library components. Our approach is illustrated through an example of a system. In fact, the dynamic model of elastic beam under the action of a moving load and carrying attached subsystems is established based on mechatronic system approach. A dynamic vibration absorber is proposed to attenuate the excessive vibration amplitude of the subsystem. A variety of numerical results are shown to draw important conclusions for the purpose of system design. This methodology can help system architects to build up rapidly system model and define solutions during the predesign phase.

\section{References}

[1] G. Hamza, M. Hammadi, M. Barkallah, J. Y. Choley, A. Riviere, J. Louati, M. Haddar, Compact analytical models for vibration analysis in Modelica/Dymola: application to the wind turbine drive train system, J. Chin. Soc. Mech. Eng. 39, 121-130 (2018)

[2] O.A. Bauchau, J. Changkuan, Modeling friction phenomena in flexible multibody dynamics, Comput. Methods Appl. Mech. Eng. 195, 6909-6924 (2006)

[3] I.I. Kosenko et al., Multibody systems dynamics: Modelica implementation and Bond Graph representation, in Proceedings of the 5th International Modelica Conference, arsenal research, Vienna, Austria, 2006, pp. 213-223

[4] A. Siemers, Contributions to Modelling and Visualization of Multibody Systems Simulations with Detailed Contact Analysis, Diss, Linköping University Electronic Press, 2010

[5] A.A. Shabana, Flexible multibody dynamics: review of past and recent developments, Multibody Syst. Dyn. 1, 189-222 (1997)

[6] I. Nakhimovski, Contributions to the modeling and simulation of mechanical systems with detailed contact analyses, Diss, Linköping University Electronic Press, 2006
[7] A. Picarelli, M. Dempsey, Investigating the multibody dynamics of the complete powertrain system, in Proceedings of the 7th International Modelica Conference; Como; Italy, Linköping University Electronic Press, 2009, pp. 423-433

[8] S. Laurent, Symbolic modeling of electromechanical multibody systems, Louvain-la-Neuve (Belgium), 2004

[9] M. Schneider, A. Haumer, R. Köckeis, Modelling and simulation of the passive structure of a 5-axis-milling machine with rigid and flexible bodies for evaluating the static and dynamic behavior, Proceedings of the 12th International Modelica Conference, Prague, Czech Republic, Linköping University Electronic Press, 2017, pp. 389-395

[10] M. Hammadi, J.Y. Choley, Parametric compact modelling of dynamical systems using meshfree method with multi-port technique, Int. J. Dyn. Syst. Diff. Eq. 5, 206-219 ( 2015)

[11] M. Hammadi, J.Y. Choley, F. Mhenni, A multi-agent methodology for multi-level modeling of mechatronic systems, Int. J. Dyn. Syst. Differ. Equ. 28, 208-217 (2014)

[12] M. Hammadi, J.Y. Choley, O. Penas, A. Riviere, J. Louati, M. Haddar, A new multi-criteria indicator for mechatronic system performance evaluation in preliminary design level, in 13th International Workshop on Mechatonics, 9th France-Japan and 7th Europe-Asia Congress on Research and Education in Mechatronics (REM), 2012, pp. 409-416

[13] R.G. Longoria, Modeling of Mechanical Systems for Mechatronics Applications, The University of Texas, Austin, USA, 2002

[14] G. Hamza, Contribution aux développements des modèles analytiques compacts pour l'analyse vibratoire des systèmes mécatroniques. Diss. Paris Saclay, 2016

[15] G. Hamza, J.Y. Choley, M. Hammadi, M. Barkallah, J. Louati, A. Riviere, M. Haddar, Analytical approach for the integrated preliminary analysis of mechatronic systems subjected to vibration, Mecatronics (MECATRONICS), 10th France-Japan/8th Europe-Asia Congress on. IEEE, 2014, pp. $151-155$

[16] G. Hamza, J.Y. Choley, M. Hammadi, M. Barkallah, J. Louati, A. Riviere, M. Haddar, Pre-dimensioning of the dynamic properties of the wind turbine system using analytical approach, Design and Modeling of Mechanical Systems-II, Springer, Cham, 2015, pp. 179-188

[17] G. Hamza, J.Y. Choley, M. Hammadi, M. Barkallah, J. Louati, A. Riviere, M. Haddar, Conceptual design methodology for the preliminary study of a mechatronic system: application to wind turbine system, Mech. Ind. 18, 413 (2017)

[18] N. Zrnić, D. Oguamanam, S. Bošnjak, Dynamics and modeling of mega quayside container cranes, FME Trans. 34, 193-198 (2006)

[19] G. Hamza, J.Y. Choley, M. Hammadi, M. Barkallah, J. Louati, A. Riviere, M. Haddar, Pre-designing of a mechatronic system using an analytical approach with dymola, J. Theor. Appl. Mech. 53, 697-710 (2015)

[20] Y.H. Lin, C.H. Cho, Vibration suppression of beam structures traversed by multiple moving loads using a damped absorber, J. Mar. Sci. Technol 1, 39-48 (1993)

[21] R.M. Soares, Z. Prado, P. Gonçalves, On the vibration control of beams using a moving absorber and subjected to moving loads, Asociación Argentina de Mecánica Computacional 29, 1829-1840 (2010) 
[22] Y.-G. Sung, Modelling and control with piezoactuators for a simply supported beam under a moving mass, J. Sound Vib. 250, 617-626 (2002)

[23] W. Gafsi, R. Chaari, N. Masmoudi, M.T. Khabou, F. Chaari, M. Haddar, Modeling of a passive absorber in milling tool machine, Appl. Acoustics 128, 94-110 (2017)

[24] F.S. Samani, F. Pellicano, A. Masoumi, Performances of dynamic vibration absorbers for beams subjected to moving loads, Nonlinear Dyn. 73, 1065-1079 (2013)

[25] M.M. Stanišić, J.C. Hardin, On the response of beams to an arbitrary number of concentrated moving masses, J. Franklin Inst. 287, 115-123 (1969)
[26] J. Fortgang, W. Singhose, Concurrent design of vibration absorbers and input shapers, J. Dyn. Syst. Measur. Control 127, 329-335 (2005)

[27] I. Esen, Dynamic response of a beam due to an accelerating moving mass using moving finite element approximation, Math. Comput. Appl. 16, 171-182 (2011)

[28] V. Gašić, N. Zrnić, A. Obradović, S. Bošnjak, Consideration of moving oscillator problem in dynamic responses of bridge cranes, FME Trans. 39, 17-24 (2011)

[29] J. Hu, E.D. Goodman, S. Li, R. Rosenberg, Automated synthesis of mechanical vibration absorbers using genetic programming, AI EDAM 22, 207-217 (2008)

Cite this article as: G. Hamza, M. Barkallah, M. Hammadi, J.-Y. Choley, A. Riviere, J. Louati, M. Haddar, Predesign of a flexible multibody system excited by moving load using a mechatronic system approach, Mechanics \& Industry 21, 604 (2020) 